\title{
Non-existence of ground states for a semilinear elliptic system of Lane-Emden-Fowler type
}

\section{MIODRAG IOVANOV}

\section{ABSTRACT.}

We obtain sufficient conditions for the non-existence of positive radially symmetric solutions for a class of Lane, Emden and Fowler elliptic systems. In our result, the nonlinear term it was suggested by the work of [D. O'Regan and H. Wang, Positive radial solutions for p-Laplacian systems, Aequationes Math., 75 (2008) 43-50].

\section{REFERENCES}

[1] Cenadelli, D., Solving the Giant Stars Problem: Theories of Stellar Evolution from The 1930s to The 1950s, Arch. Hist. Exact Sci., 64 (2010), 203-267

[2] Chandrasekhar, S., An introduction to the study of stellar structure, Dover Publications, Inc., New York, N. Y. 1957

[3] Chandrasekhar, S., On Stars, Their Evolution and Their Stability, Nobel lecture, 8 December, 1983

[4] Cîrstea, F. -C. Şt. and Rădulescu, V. D., Existence and uniqueness of positive solutions to a semilinear elliptic problem in $\mathbb{R}^{N}$, J. Math. Anal. Appl., 229 (1999), No. 2, 417-425

[5] Covei, D. -P., Non-existence criteria for solutions of the Lane-Emden-Fowler system, Appl. Math. Lett., 25 (2012), No. 3, 610-613

[6] Covei, D.-P., Existence results for a quasilinear elliptic problem with a gradient term via shooting method, Appl. Math. Comput., 218 (2011), No. 8, 4161-4168

[7] Covei, D. -P., A Lane-Emden-Fowler type problem with singular nonlinearity, J. Math. Kyoto Univ., 49 (2009), No. 2, 325-338

[8] Covei, D. -P., Non-existence result for radially symmetric solutions to the Lane-Emden-Fowler equations, Nonlinear Anal., Theory Methods Appl., 70 (2009), No. 1-A, 563-566

[9] Covei, D. -P., Sufficient conditions for the existence and asymptotic behavior of solution to a quasilinear elliptic problem, Int. J. Comput Math., 88 (2011), No. 12, 2637-2647

[10] Diaz, J. I., Nonlinear Partial Differential Equations and Free Boundaries, Pitman Research Notes in Mathematics, 106,1985

[11] Ghergu, M., Lane-Emden systems with negative exponents, J. Funct. Anal., 258 (2010), No. 10, 3295-3318

[12] Goncalves, J. V. and Silva, F. K., Existence and nonexistence of ground state solutions for elliptic equations with a convection term, Nonlinear Anal., Theory Methods Appl., Ser. A, Theory Methods, 72 (2010), No. 2, 904-915

[13] Goncalves, J. V. and Silva, F. K., Solutions of quasilinear elliptic equations in $R^{N}$ decaying at infinity to a nonnegative number, Complex Var. Elliptic Equ., 55 (2010), No. 5-6, 549-571

[14] Gonçalves, J. V., Rezende, M. C. and Santos, C. A., Positive solutions for a mixed and singular quasilinear problem, Nonlinear Anal., Theory Methods Appl., Ser. A, Theory Methods, 74 (2011), No. 1, 132-140

[15] Garcia-Huidobro, M. and Manasevich, R., Existence of ground states for quasilinear nonhomogeneous elliptic systems, J. Math. Anal. Appl., 251 (2000), 669-694

[16] Ladde, G. S., Lakshmikantham, V. and Vatsale, A. S., Existence of coupled quasi-solutions of systems of nonlinear elliptic boundary value problems, Nonlinear Anal., Theory Methods Appl, 8 (1984), 501-515

[17] Lair, A. V. and Shaker, A. W., Entire solution of a singular semilinear elliptic problem, J. Math. Anal. Appl., 200 (1996), No. 2, 498-505

[18] Lazer, A. C. and McKenna, P. J., On a singular nonlinear elliptic boundary value problem, Proc Amer. Math. Soc., 111 (1991), 721-730

Received: 07.02.2012; In revised form: 03.07.2012; Accepted: 14.09.2012

2010 Mathematics Subject Classification. 34A30, 34A34.

Key words and phrases. Non-Existence, Lane-Emden-Fowler System. 
[19] Leung, A. and Fan, G., Existence of positive solutions for elliptic systems-degenerate and nondegenerate ecological models, J. Math. Anal. Appl., 151 (1990), No. 2, 512-531

[20] O'Regan, D. and Wang, H., Positive radial solutions for p-Laplacian systems, Aequationes Math., 75 (2008), 43-50

[21] Usami, H., Positive solutions of singular Emden-Fowler type systems, Hiroshima Math. J., 22 (1992), No. 2, $421-431$

[22] Zhang, Z, Positive solutions of Lane-Emden systems with negative exponents: Existence, boundary behavior and uniqueness, Nonlinear Anal., Theory Methods Appl., Ser. A, Theory Methods, 74 (2011), No. 16, 5544-5553

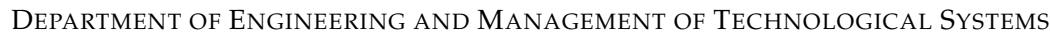

CONSTANTIN BRANCUSI UNIVERSITY OF TG-JIU

EROILOR 30, 210135 TG-JIU, ROMANIA

E-mail address: iovanovmeyahoo.com 